JASC 12-1-7

\title{
Design of Korean smart car driving information checking system
}

\author{
Min young Kim ${ }^{\dagger}$, Jong Wook Jang* \\ ${ }^{\dagger}$ Department of Computing Engineering, Dong-eui University, Korea
}

\begin{abstract}
Automobilist in South Korea in order to provide car driving information to the insurance company uses 'car driving information device'. They provide car driving information of device to insurers for their premium discount. Currently these devices are used in Korea has inconvenience that this device Installation and the process for send this information to insurance companies.

In this paper, Korean smart car driving information system designed using Smart phone and local-range network as a basis the 'Certification Regulations of Driving Information Check Device' of the Korea Insurance Development Institute.
\end{abstract}

Key words : OBD protocol, No-driving day car insurance, Driving information check device, Driving information collection system

\section{INTRODUCTION}

From June 2010, Korea's car insurance companies carried out a no-driving day car insurance, which is a special contract system under the terms of the contracts that the driver can choose a single day among from Monday to Friday and has not to drive a car every selected single same day, and that if the driver keeps in touch with it during the period of buying that insurance, the company has to give a discount of about $8.7 \%$ of the insurance price to the insurer. The Korea Insurance Development Institute introduced this special contract system of car insurance in Korea because some foreign countries such as the United States, Germany, Japan conducted this special insurance system earlier than Korea and received a good response from the insurers after changing some items of the contracts to meet each country's discount ratio system.

In Korea, if you want to buy this special no-driving day car insurance, you have to follow two conditions after enrolling the insurance company; First, you should purchase a personal Driving Information Check Device (OBD terminal) to attach it to your car; and Finally, you should enroll in the Driving Information Collection System of the company via internet

*Corresponding Author: jwjang@deu.ac.kr

Tel: +82-51-890-1709, Dong-eui Univ.

*Dept. of Computing Engineering, Dong-eui Univ., Korea
[1].

The currently available personal Driving Information Check Device attached to the car's OBD port can record your driving information, and the stored driving information can be sent to the Driving Information Collection System of the company through the company's homepage logged in after detaching the device from OBD port and connecting it with USB of the PC. In this processing, whenever sending the driving record information to the company, the insurer felt uncomfortable in that (s)he must attach the device to the car's OBD port and again detach it from that port.

In this paper, in order to solve those problems of the existing personal Driving Information Check Device, a newly designed personal Smartphone Vehicle Driving Information Check System using a local-range wireless communication and a smartphone will be suggested with the contents. Based on the 'Certification Regulations of Driving Information Check Device' of the Korea Insurance Development Institute, a hardware design for personal Vehicle Driving Information Check Device was studied; and a smartphone multifunctional terminal monitor program, that can directly send the driving record information to the Car Driving Information Collection System of the insurance company, was studied as well.

\section{OVERALL SYSTEM CONFIGURATION}

A. Existing System Configuration 
The existing system has to attach a Driving Information Check Device to the car's OBD port. From that moment, the check device can store the car's driving record by extracting and calculating the necessary information through OBD protocols with the ECU's information. After a given constant interval, the insurer have to obey the following steps; first, detach the Driving Information Check Device from the car's OBD port; second, connect the check device with the PC by using an USB; third, make the driving information record encrypted files by using the terminal monitor program; fourth, find the corresponding car insurance company homepage and $\log$ in it in order to enter and use the Driving Information Upload Web Applications of the company; and finally, send the encrypted files to the Driving Information Collection System(a server) of the company.

The insurance company, which receives the driving record from the insurer, has to obey the following steps; first of all, check whether the insurer drove a car every selected single same day or not; second, if (s)he did not drive on that day all the time, the company has to give a discount to the insurer; and finally, the company has to determine how much to give a discount to the insurer. ("Fig 1")

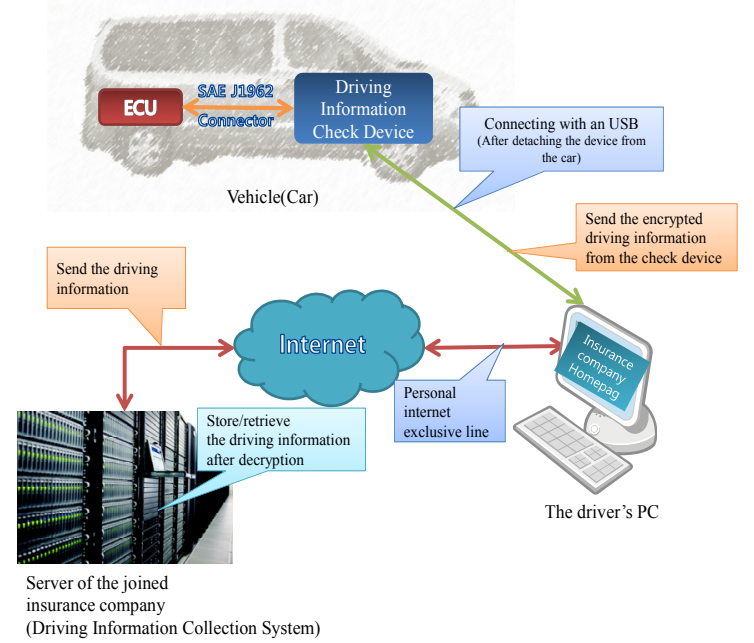

Fig 1. The existing overall system configuration

\section{B. Proposed Overall System Configuration}

Whenever the insurer has to send the driving information of the Driving Information Check Device to the corresponding insurance company, (s)he can feel inconvenient because of the burden such as attaching and detaching the check device as well as using a PC. In addition, if the processes such as attaching and detaching the check device are continuously repeated, the economic burden could be occurred when the check device damaged the internal circuits or had bad appearance by the chocks during the processes.
In this paper, in order to solve such problems, a Bluetooth or Wi-Fi module among the local-range wireless communications can be selected to add the existing Driving Information Check Device in addition to the USB connection method that is selected in the existing Driving Information Check Device of the insurer. This is possible because the driving information can be fetched from the Driving Information Check Device through a built-in local-range wireless communication module in the smartphone in the Smartphone Terminal Monitor Program. The Terminal Monitor Program can send the driving information fetched from this device via $3 \mathrm{G}$ or Wi-Fi wireless communication network after the information backed up into the smartphone to the Driving Information Collection System of the insurance company ("Fig 2"). If it is designed such a method, problems rising in the existing system configuration, for example, problems occurring during the installation and use of the check device, can be solved.

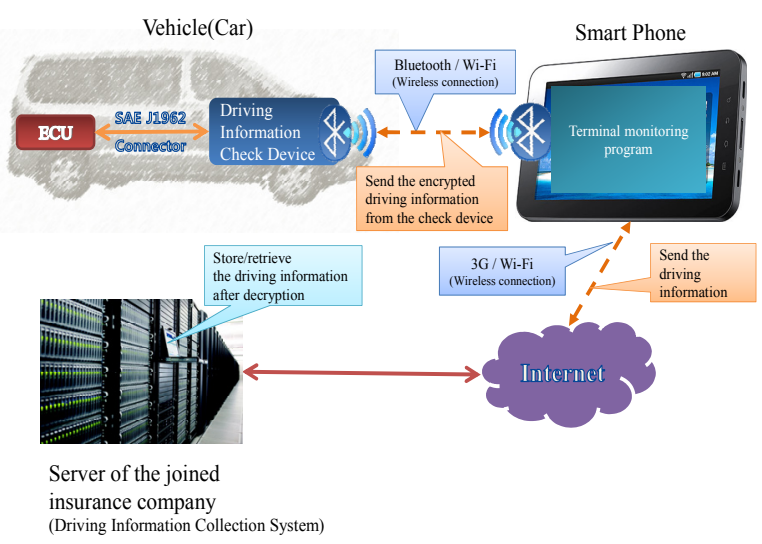

Fig 2. The proposed overall system configuration

\section{OVERALL SYSTEM CONFIGURATION}

\section{A. Hardware Design}

In order to meet the standards of the Korea Insurance Development Institute, a port that can be connected with the car's OBD, an arithmetic unit that can extract and calculate various information by using $\mathrm{OBD}$ communication protocols, an non-volatile memory that can store the encrypted driving information records, a real-time clock that can check the exact time, an built-in battery and a connection way(wire and wireless connections) that can send the driving information should be included in the hardware design. In addition, in order to meet the condition that is compatible with other vehicle communication standards that do not have the OBD-II standards, a means to identify the conversion gender and terminal operation status should be provided in the design [2]. 
For this hardware design, to meet the standards of the Korea Insurance Development Institute, also the J1962 connector to connect with an OBD port, an 32-bit Micro Controller (arithmetic unit) to process the driving records and various information fast and exactly, a Transceiver to use the vehicle communication standards (CAN, KWP-2000, J1850) that makes the arithmetic unit use the OBD protocol, a Flash Memory to store the driving records, a Timer to check the exact time, a Bluetooth or Wi-Fi module to send the driving information to a PC and a smartphone, and a speaker and high-brightness LED to identify the operation status of the terminal should be built in this design("Fig 3"). The additionally attached GPS module can be built-in to compensate the time by using the Greenwich Mean Time.

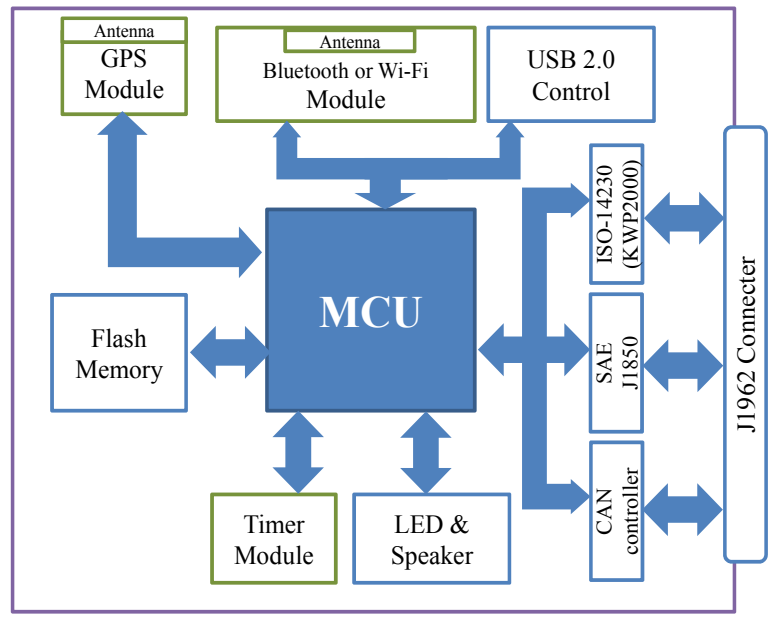

Fig 3. Hardware configuration [3]

\section{B. Design of hardware Firmware}

Among the proposed hardware design, in the internal EPPROM of the Micro Controller, there exists built-in firmware, which is essential part of the Driving Information Device.

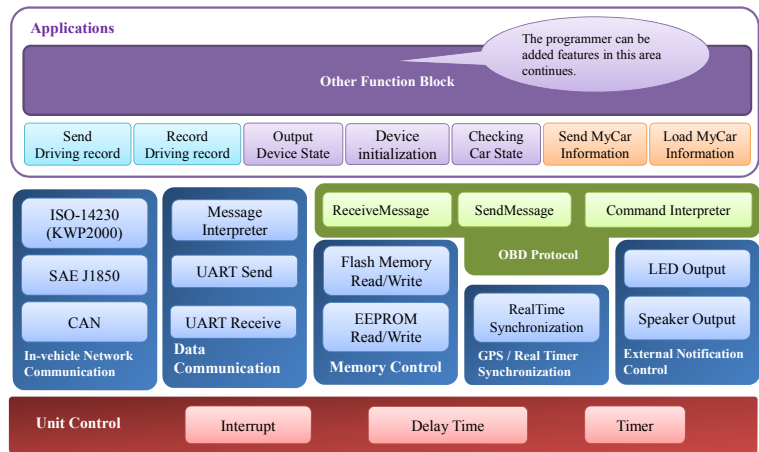

Fig 4. Configuration of Firmware Framework

"Fig 4" shows the configuration of the firmware framework to be used in this hardware design. This framework was divided into several modules with their own functions. Later, in addition to the basic functions, if any functions can be added, they are added into the Applications module.

The proposed firmware framework, which was divided into several modules by classifying how often the functions were used by types, consists of Unit Control, In-Vehicle Network Communication, Data Communication, Memory Control, GPS/Real Synchronization, External Notification Control, OBD Protocol and Base Applications modules. In the configuration of "Fig 4", each module, which has the hierarchical relationship with the corresponding modules of the upper layer and just below layer, is designed to carry out instructions by receiving functions from the corresponding module of the upper layer and using functions in the corresponding module of the just below layer.

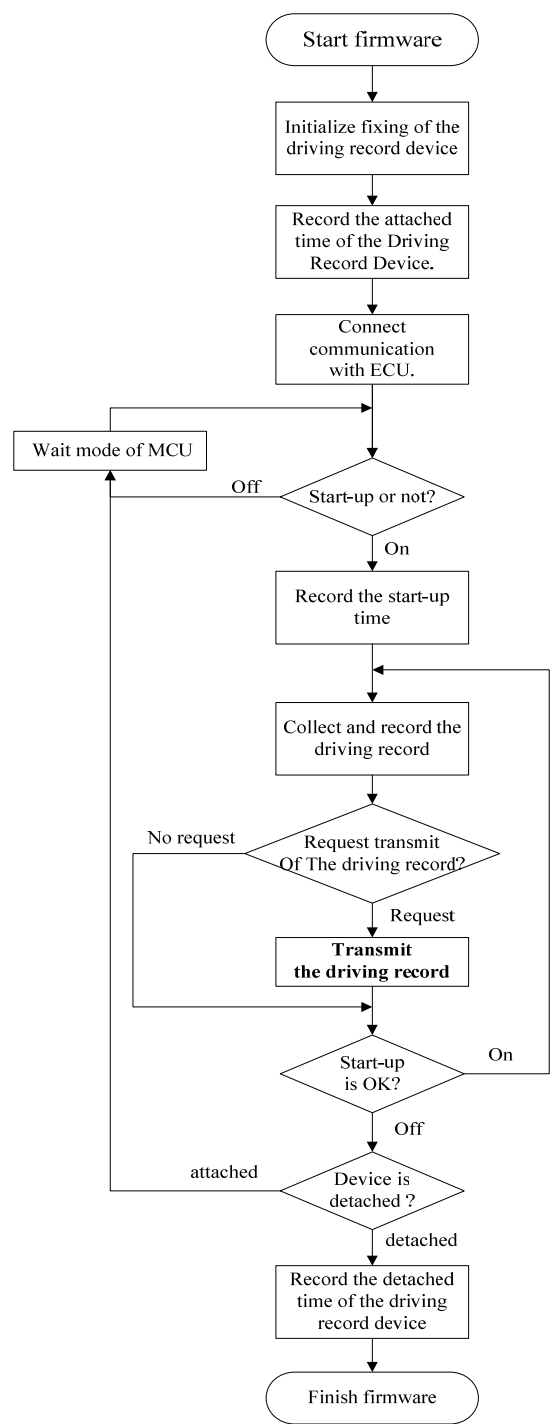

Fig 5. Flowchart of firmware execution 
"Fig 5" shows a flowchart of the firmware. First of all, in case of attaching a Driving Information Check Device to the car, plug in the car's battery and then carry out to set up the Driving Record Device. After that, setting up the MCU's initialization, communication with the internal ECU and Bluetooth or Wi-Fi communication, and performance time correction by using the Greenwich Mean Time after receiving the GPS information can be designed.

Based on the standards of the Korea Institute Development Institute, the requested information (the driving distance, the accelerator pedal, the throttle valve open angle and etc.) can be extracted from the necessary information received from the ECU by using OBD protocols to the vehicle network. After extracting the requested information, the user has to encrypt the recorded driving information in order not to be changed and then store it into the Flash Memory of the terminal.

In order to follow the standards of the Korea Insurance Development Institute, it was designed to all record the device's attach/detach time, start-up time, finish time, driving start/finish time as well as the vehicle identification number[2].

In the firmware, in order to process easily the driving information record received from the Terminal Monitor Program, the records and fields of the files can be designed to follow the standards.

\section{SMART TERMINAL MONITOR PROGRAM}

The Smart Terminal Monitor Program in this paper can be designed to be used in the operating system/platform of a smartphone or smartpad, and to provide easy functions and user-friendly interfaces in order to make the user identify the driving information ("Table 1").

Table 1. The availability of each OS/Platform Local-range wireless communications

\begin{tabular}{r|cc}
\hline Communication type & Wi-Fi Ad-hoc & Bluetooth \\
\hline Ondrotform & $\triangle$ & $\bigcirc$ \\
iOS $^{2}$ & $\bigcirc$ & $\times$ \\
WindowsPhone & $\times$ & $\times$ \\
\hline
\end{tabular}

The proposed Driving Information Check Device in this paper includes key functions such as driving information check, terminal/vehicle(or ECU) identification number check, driving information backup and the insurer's driving information sending to the Driving Information Collection

\footnotetext{
${ }^{1}$ Android 4.0(ICS) versions support the Ad-hoc mode.

${ }^{2}$ IPhone Bluetooth devices must be certified by Apple (MFI License)
}

System of the insurance company[2].

The Terminal Monitor Program for a smartphone can store the encrypted driving information from the Driving Information Check Device into the backup DB, and if it sends the information through the backup DB to the user according to the functions listed above, the corresponding information in the DB can be sent to the user after running the decryption process of the information ("Fig 6").

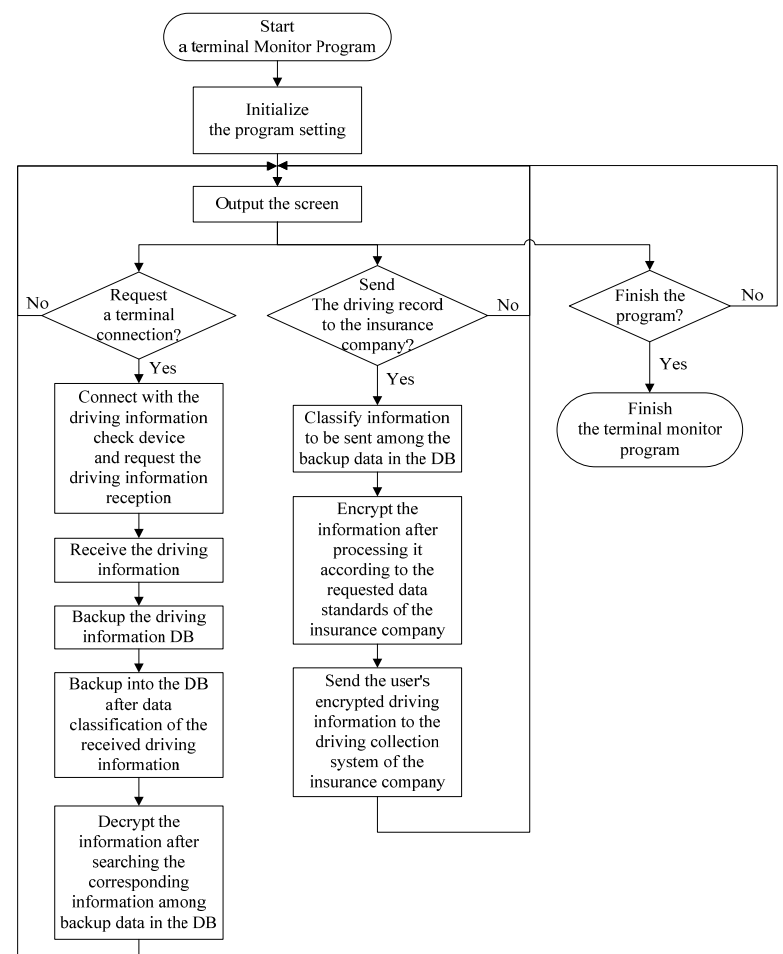

Fig 6. Flowchart of smart phone terminal monitor program

When the insurer's driving record is to be sent to the Driving Information Collection System of the corresponding insurance company, it has to be sent to the company after applying the encryption algorithm and transmission information protocols provided by the company to it and processing it properly, and the setup part of the Terminal Monitor Program should include functions that can save the company's server address and the user's information so that the driving record can be sent to the Driving Information Collection System of the company fast and exactly after logging in the company's homepage("Fig 6").

Also, in consideration of the characters of a smartphone, in the resource-poor embedded systems, to utilize the resources, APIs that process SQL instructions in the DBMS do not be provided. In a smartphone, however, it is possible to send and receive data in XML, so that functions of sending and receiving XML documents can be provided in the Driving Information Collection System of the insurance company. 


\section{CONCLUSION AND CONSIDERATION}

In this paper, the proposed Smartphone Driving Information Check System for a no-driving day car insurance, in order to solve some problems rising during the installation and use of the existing Driving Information Check Device on the car, includes a local-range wireless communication such as Wi-Fi Ad-hoc or Bluetooth to send the insurer's driving information to the Smartphone's Terminal Monitor Program, backup the driving record and finally send the driving record to the Driving Information Collection System of the insurance company.

In the near future, a vehicle with EDR (Event Data Record), which can save several situations rising during car accidents with the information based on the standards of the Korea Insurance Development Institute and offer available information as well, should be developed, and also, for a smartphone, a Vehicle Check Monitoring Program, which can check all status of the user's car at the same time by using the contents of the EDR, should be designed and implemented.

\section{ACKNOWLEDGMENT}

This research was financially supported by the Ministry of Education, Science Technology (MEST) and National Research Foundation of Korea (NRF) through the Human Resource Training Project for Regional Innovation

\section{REFERENCES}

[1] The Korea Insurance Development Institute: http://www.kidi.or.kr/.

[2] The Korea Insurance Development Institute: "Performance Test Standards of a driving information check device for a no-driving day car insurance", Nov. 2009

[3] O-Sup Son: "study for development of power-saving vehicle diagnostics module hardware based on OBD-II", pp.34, Thesis of Engineering Master degree, Dong-Eui University, Feb. 2011

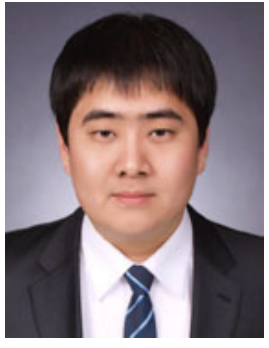

Min Young Kim was born in Busan, Korea in 1986. He received the B.S. degrees in Computer engineering from University of the Dong-eui in 2011. Since 2011, he is currently a Master students in Computer engineering University of the Dong-eui. His research interests are in the areas of Vehicle Network, WPAN, and Software Engineering. Mr. Kim is a Member of the Institute of Webcasting, Internet and Telecommunication (IWIT) of Korea.

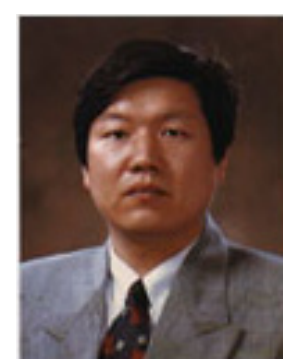

Jong Wook Jang was born in Busan, Korea, in 1961. He received the B.S., M.S. and Ph.D. degrees in Computer Science and Computer engineering from the University of the Busan, Chungnam, Busan in 1987, 1991 and 1995 respectively. Since 1995, he has been with the Department of Computer engineering of Dong-eui University, where he is currently a professor. His research interests are in the areas of Wired and wireless communication systems, and Vehicle Network. Prof. Jang is a member of the Institute of Webcasting, Internet and Telecommunication(IWIT) of Korea. 